\title{
EFICIENCIA AGRONÓMICA DE NITRÓGENO Y PRODUCCIÓN DE Cynodon plectostachyus (K. Schum.) Pilg. EN FUNCIÓN DE DOS FRECUENCIAS DE CORTE
}

\section{AGRONOMIC EFFICIENCY OF NITROGEN AND PRODUCTION OF Cynodon plectostachyus (K. Schum.) Pilg. DEPENDING ON TWO CUTTING FREQUENCIES}

\author{
Dídimo Vera Arteaga ${ }^{1 \dagger}$, Galo Cedeño García ${ }^{2 *}$, George Cedeño-García ${ }^{3}$, Jessica Cargua Chávez ${ }^{4}$, y \\ María Garay Lugo ${ }^{1}$
}

${ }^{1}$ Departamento de Producción Animal, Facultad de Ciencias Zootécnicas, Universidad Técnica de Manabí, km $2 \frac{1}{2} 2$ vía Chone - Boyacá, Chone, Manabí, Ecuador.

${ }^{2}$ Carrera de Ingeniería Agrícola, Escuela Superior Politécnica Agropecuaria de Manabí Manuel Félix López, Campus Politécnico El Limón, km 2 1⁄2 vía Calceta - El Gramal. Calceta, Manabí, Ecuador.

${ }^{3}$ Departamento de Agronomía, Facultad de Ingeniería Agronómica, Universidad Técnica de Manabí, km 13 1⁄2 vía Portoviejo - Santa Ana, Santa Ana, Manabí, Ecuador.

${ }^{4}$ Carrera de Tecnología Superior en Producción Agrícola, Calle B, Santo Domingo de los Tsáchilas, Ecuador.

*Autor para correspondencia: gcedeno@espam.edu.ec

${ }^{\dagger}$ Fallecido durante proceso de investigación

\section{RESUMEN}

La fertilización nitrogenada y la frecuencia de corte son imprescindibles para mejorar la producción y calidad nutritiva del forraje. El objetivo de la investigación fue evaluar la eficiencia agronómica de $\mathbf{N}$ y rendimiento del pasto estrella Cynodon plectostachyus en función de dos frecuencias de corte bajo condiciones de trópico bajo. Los tratamientos consistieron en cinco niveles de $N(0,50,100,150$ y $\left.200 \mathrm{~kg} \mathrm{ha}^{-1}\right)$ y dos frecuencias de corte (30 y 45 días). Las variables evaluadas fueron rendimiento de materia seca (MS) y eficiencia agronómica del N (EAN) aplicado. El rendimiento de MS y la EAN fueron influenciadas significativamente $(p<0,01)$ por los niveles de $N$, frecuencias de corte y la respectiva interacción, excepto para EAN donde la interacción no fue significativa $(p>0,05)$. El rendimiento de MS y EAN mostraron un comportamiento lineal con el incremento de $\mathrm{N}$, independientemente de las frecuencias de corte. Independientemente de los niveles de $\mathrm{N}$, el rendimiento de MS y EAN se incrementaron con la frecuencia de corte más amplia. La MS se incrementó en 8,39; 14,40; 20,14 y $26,24 \%$ con 50, 100, 150 y $200 \mathrm{~kg} \mathrm{~N} \mathrm{ha}^{-1}$, respectivamente, con respecto a la parcela con omisión de $\mathrm{N}$. La EAN en promedio fue de 2,72; 3,35; 3,59 y 4,18 $\mathrm{kg}^{\mathrm{de}} \mathrm{MS} \mathrm{kg} \mathrm{kg}^{-1} \mathrm{de} \mathrm{N}$ aplicado con 50, 100, 150 y 200 $\mathrm{kg} \mathrm{N} \mathrm{ha}^{-1}$, respectivamente, aunque los niveles de 150 y 200 fueron estadísticamente iguales. La dosis de $150 \mathrm{~kg}$ de $\mathrm{N} \mathrm{ha}^{-1}$ sería suficiente para la fertilización del pasto.

Palabras clave: Pasto estrella, Cynodon plectostachyus, nitrógeno, nutrición, fertilización, uso eficiente

\section{ABSTRACT}

Nitrogen fertilization and cutting frequency are essential to improve the production and nutritional quality of forage crops. The objective of this study was to evaluate the agronomic efficiency of

Recibido: 27 mayo 2019. $\quad$ Aceptado: 24 junio 2019. 
$\mathrm{N}$ and performance of the Cynodon plectostachyus star grass based on two cutoff frequencies under low tropic conditions. The treatments consisted of five levels of $N\left(0,50,100,150\right.$ and $\left.200 \mathrm{~kg} \mathrm{ha}^{-1}\right)$ and two cutoff frequencies ( 30 and 45 days). The variables evaluated were dry matter yield (DM) and agronomic efficiency of $\mathrm{N}$ (AEN) application. The production of DM and AEN were significantly influenced $(p<0.01)$ by the levels of $N$, cutoff frequencies and the respective interaction, except for AEN where the interaction was not significant $(p>0.05)$. DM and AEN showed a linear behavior with an increase in $\mathrm{N}$, regardless of the cutoff frequencies. Similarly, DM and AEN performance increased with the higher cutoff frequency. DM increased by $8.39 ; 14.40 ; 20.14$ and $26.24 \%$ with $50,100,150$ and $200 \mathrm{~kg} \mathrm{~N} \mathrm{ha}^{-1}$, respectively, with respect to the plot with no $\mathrm{N}$ application. The average AEN was 2.72; $3.35 ; 3.59$ and $4.18 \mathrm{~kg}$ of DM kg-1 of $\mathrm{N}$ applied with 50, 100, 150 and $200 \mathrm{~kg} \mathrm{~N} \mathrm{ha}^{-1}$, respectively. However, levels of 150 and 200 were statistically equal. The dose of $150 \mathrm{~kg}$ of $\mathrm{N} \mathrm{ha}^{-1}$ would be sufficient for grass fertilization.

Key words: Star grass, Cynodon plectostachyus, nitrogen, nutrition, fertilization, efficient use

\section{INTRODUCCIÓN}

En Ecuador se estima actualmente una superficie dedicada a pastos de 3.087 .780 hectáreas, de las cuales el $73 \%$ corresponden a pastos cultivados. Del total nacional, el $56,64 \%$ se encuentra en la región costa, donde la provincia de Manabí posee la mayor área cultivada, siendo el cantón Chone el mayor productor (MAGAP, 2016; Bonifaz et al., 2018). El pasto más predominante en el país es el guinea (Megathyrsus maximus) con 1.147.091 ha, que es apreciado por su resistencia a sequía, sin embargo, en zonas bajas puede encontrase hasta un 5\% del área con especies de pasto estrella Cynodon plectostachyus y C. nlemfluensis, de las cuales existe escasa o nula información sobre su potencial productivo y respuesta a la fertilización (Castillo, 2014; Bonifaz et al., 2018).

En estudios realizados en zonas tropicales y subtropicales se ha encontrado que el pasto estrella presenta buen potencial de crecimiento, rendimiento y calidad nutritiva para la producción láctea, manejando los días adecuados de rotación y recuperación en conjunto con la fertilización, para optimizar su rendimiento, calidad y persistencia (López et al., 2010; Yong et al., 2012; Villalobos y Arce, 2013; 2014). Las especies de pasto Cynodon son de metabolismo fotosintético $\mathrm{C}_{4^{\prime}}$ por lo que pueden acumular mayor materia seca en relación a los pastos $C_{3}$ se cultivan ampliamente en zonas tropicales y subtropicales, y responden a la fertilización nitrogenada para incrementar el rendimiento y calidad nutritiva (Yong et al., 2014; Cristiano et al., 2015; Taffarel et al., 2016; Borges et al., 2017; Schneider et al., 2019).

Sin embargo, el uso excesivo de fertilizantes nitrogenados en el suelo puede repercutir en una acumulación excesiva de nitratos en el forraje, que es la principal fuente de intoxicación por nitrato en los animales lecheros (Rashid et al., 2018). En este contexto, Barhoumi (2017) encontró que una alta disponibilidad de $\mathrm{N}$ incrementó 3 y 20 veces la acumulación de nitrato en brotes y raíces de Brachypodium distachyon; por otra parte Rashid et al. (2018) encontraron contenidos mayores a 5000 $\mathrm{mg} \mathrm{kg}^{-1}$ de nitrato en tallos de cultivos forrajeros y como consecuencia altos niveles de nitrito en sangre de animales que consumieron los forrajes testeados.

Es importante destacar que un exceso de $\mathrm{N}$ en el sistema de producción contribuye a la contaminación del ambiente, especialmente en los trópicos, donde la eficiencia del nitrógeno de los fertilizantes es baja; aproximadamente un tercio del fertilizante nitrogenado aplicado se pierde en la atmósfera o se filtra hacia aguas subterráneas en forma de amoniaco, óxido nitroso y nitratos; por tanto, se ha sugerido el uso de varias estrategias para mejorar la eficiencia de la fertilización nitrogenada y reducir las pérdidas de N en el ambiente (Prasad, 2013; Sharma and Bali, 2017; Yadav et al., 2017; Zhao et al. 2019).

Actualmente se vienen desarrollando estrategias para reducir las pérdidas de $\mathrm{N}$ e incrementar su uso eficiente en los cultivos (Yadav et al., 2017). Entre las principales estrategias destaca el desarrollo de genotipos con mayor eficiencia de uso del $\mathrm{N}$ proveniente de la fertilización, dado que los complejos procesos bioquímicos, fisiológicos y moleculares implicados en la absorción y metabolismo del $\mathrm{N}$ están influenciados genéticamente (Han et al., 2015; Cormier et al., 2016). Otra de las estrategias es el uso de fuentes nitrogenadas de eficiencia mejorada, que son fertilizantes que reducen las diversas pérdidas asociadas con el sistema de producción. Estos fertilizantes se basan en dos filosofías: pueden disminuir la velocidad de liberación del nutriente o pueden interferir con los procesos de transformación y reducir sus pérdidas. Los fertilizantes nitrogenados 
de liberación controlada y los inhibidores de nitrógeno son dos clases importantes de estas fuentes (Li et al., 2017; Yadav et al., 2017; Mohan et al., 2018). Por lo tanto, la respuesta de los cultivos al nitrógeno aplicado y su eficiencia de uso deben monitorearse continuamente como estrategia para maximizar el potencial de rendimiento por cada unidad de $\mathrm{N}$ utilizado en el sistema de producción (Hirel et al., 2011; Rütting et al., 2018). En este sentido, recientes investigaciones de fertilización nitrogenada en pasto Cynodon en relación con la frecuencia de defoliación y riego del pasto, han demostrado que estos estudios son imprescindibles para optimizar la eficiencia de uso del $\mathrm{N}$ y establecer tiempos óptimos de consumo para rumiantes, y así aprovechar al máximo la producción y calidad nutritiva de la pastura (Taffarel et al., 2016; Borges et al., 2017; Sanches et al., 2017).

Establecer la frecuencia de corte o defoliación en pastos tropicales tiene importancia debido a que la edad afecta la producción, calidad y digestibilidad de la pastura, dado el incremento de fibra, lignina, tallos y material muerto a medida que la planta madura (Valles et al., 2016). En este sentido estudios realizados en Brasil por Pereira et al. (2011) y Taffarel et al. (2016) encontraron que la frecuencia de defoliación afecta la calidad y la morfogénesis de Cynodon spp. cv. Tifton 85, donde el corte de 28 días después de la defoliación mostró mejores valores bromatológicos, mientras que a los 35 días de la defoliación la producción se incrementó, luego de este tiempo la relación hoja-tallo disminuye, debido a una reducción en la tasa de emisión de hojas, aumento del filocrón, senescencia de hojas y muerte.

La eficiencia de uso de nutrientes es un término dinámico y complejo que incluye una gama de componentes, que reflejan la recuperación de nutrientes, balance o rendimiento producido por unidad de nutriente aplicado (IPNI, 2012). El índice más utilizado por investigadores para evaluar el uso eficiente de nutrientes es la eficiencia agronómica (EA) que indica las unidades de incremento en rendimiento por unidad de nutriente aplicado. Para el cálculo de EA esencialmente se requiere el establecimiento de una parcela de omisión del nutriente estudiado (IPNI, 2012; Yadav et al., 2017).

En la zona de estudio se desconoce la eficiencia de la fertilización nitrogenada en el pasto $C$. plectostachyus, por lo que se supuso que el pasto al ser una especie $\mathrm{C}_{4}$ respondería eficientemente a la fertilización nitrogenada, considerando los estudios reportados por la literatura reciente en países tropicales. Por lo anterior, el objetivo de la investigación fue evaluar la eficiencia agronómica de $\mathrm{N}$ y rendimiento del pasto $\mathrm{C}$. plectostachyus en función de dos frecuencias de corte bajo condiciones de trópico bajo.

\section{MATERIALES Y MÉTODOS}

La investigación se desarrolló durante la temporada seca (julio-diciembre) del año 2017 con una duración de 180 días, en la comunidad La Margarita $\left(0^{\circ} 41^{\prime} 18^{\prime \prime} \mathrm{S}\right.$; $\left.80^{\circ} 07^{\prime} 26^{\prime \prime} \mathrm{O}\right)$ ubicada en el trópico bajo del cantón Chone, provincia de Manabí, Ecuador, a una altitud de $5 \mathrm{msnm}$. El clima local es clasificado como Herbazal inundado lacustre del Pacífico Ecuatorial (HsTc05), de acuerdo al sistema de clasificación de ecosistemas del Ecuador continental (MAE, 2013). Las temperaturas oscilan entre $20 \mathrm{y}$ $31,8^{\circ} \mathrm{C}$ con promedio anual de $27,7^{\circ} \mathrm{C}$, y las precipitaciones varían entre $800-1200 \mathrm{~mm}$ (MAE, 2013). El suelo de la zona es clasificado como Fluventic Eutrudepts y se caracteriza por tener la siguiente secuencia de horizonte: $\mathrm{A} / \mathrm{Bw} / \mathrm{BC} / \mathrm{C}$, textura arcillosa, buen drenaje natural, $\mathrm{pH}$ de 6,6 y una profundidad efectiva de $70 \mathrm{~cm}$ (Moreno et al., 2018). El experimento se desarrolló en praderas previamente establecidas con el pasto C. plectostachyus, a las cuales se les practicó un corte uniforme de igualación a $5 \mathrm{~cm}$ por encima del nivel del suelo con ayuda de una cortadora mecánica (Taffarel et al., 2016).

El experimento se estableció en un diseño de bloques al azar con tratamientos factoriales de cinco niveles de fertilización con $\mathrm{N}(0,50,100$, 150 y $\left.200 \mathrm{~kg} \mathrm{ha}^{-1}\right)$ y dos frecuencias de corte (30 y 45 días después del corte DDC), con cuatro réplicas y 40 unidades experimentales. La unidad experimental se conformó de parcelas de $5 \times 5 \mathrm{~m}$ $\left(25 \mathrm{~m}^{2}\right)$. Para una mayor precisión y consistencia de resultados, se realizaron cuatro ciclos de corte de 30 y 45 días. Las parcelas con una frecuencia de corte de 30 días se cosecharon el 30 de julio, el 29 de agosto, el 28 de septiembre, y 28 de octubre de 2017. Las parcelas con una frecuencia de corte de 45 días se cortaron el 14 de agosto, el 21 de septiembre, el 5 de octubre y el 19 de noviembre de 2017. El pasto se regó semanalmente mediante riego presurizado de aspersión. Como fuente de $\mathrm{N}$ se utilizó urea $(46 \% \mathrm{~N})$, que fue aplicada dentro de los cinco días posteriores después del corte inicial y luego de cada uno de los cortes posteriores. No se aplicó fertilización fosfórica y potásica debido a los altos niveles reportados por el análisis químico de suelo (Tabla 1).

Para el registro de variables se utilizó un cuadrante de PVC de $1 \mathrm{~m}^{2}$, con el cual se realizaron tres muestreos al azar en el centro de la unidad experimental para ambas frecuencias de corte ( 30 y 45 días). La principal variable de rendimiento evaluada fue materia seca (MS) en $\mathrm{kg} \mathrm{ha}^{-1}$; para 
Tabla 1. Análisis químico de suelo realizado en el ensayo experimental previo a la aplicación de tratamientos. La muestra de suelo fue obtenida a una profundidad de $0-20 \mathrm{~cm}$.

Table 1. Chemical analysis of the soil carried out in the experimental test prior to the application of treatments. The soil sample was taken at a depth of $0-20 \mathrm{~cm}$.

\begin{tabular}{|c|c|c|c|c|c|c|c|}
\hline \multirow{2}{*}{ Nutriente } & \multirow{2}{*}{ Unidad } & \multicolumn{3}{|c|}{ Valores de referencia } & \multicolumn{3}{|c|}{ Resultados } \\
\hline & & Bajo & Medio & Alto & Bajo & Medio & Alto \\
\hline $\mathrm{N}-\mathrm{NH}_{4}{ }^{1 /}$ & $\mathrm{mg} \mathrm{kg}^{-1}$ & $<20$ & $20-40$ & $>40$ & 4 & & \\
\hline $\mathrm{P}^{1 /}$ & $\mathrm{mg} \mathrm{kg}^{-1}$ & $<10$ & $10-20$ & $>20$ & & & 38 \\
\hline $\mathrm{S}^{2 /}$ & $\mathrm{mg} \mathrm{kg}^{-1}$ & $<10$ & $10-20$ & $>20$ & & 18 & \\
\hline $\mathrm{K}^{1 /}$ & meq $100 \mathrm{~mL}^{-1}$ & $<0,2$ & $0,2-0,4$ & $>0,4$ & & & 0,89 \\
\hline $\mathrm{Ca}^{1 /}$ & meq $100 \mathrm{~mL}^{-1}$ & $<4$ & $4-8$ & $>8$ & & & 17 \\
\hline $\mathrm{Mg}^{1 /}$ & meq $100 \mathrm{~mL}^{-1}$ & $<1$ & $1-2$ & $>2$ & & & 3,3 \\
\hline $\mathrm{Cu}^{1 /}$ & $\mathrm{mg} \mathrm{kg}^{-1}$ & $<1$ & $1-4$ & $>4$ & & & 11 \\
\hline $\mathrm{Fe}^{1 /}$ & $\mathrm{mg} \mathrm{kg}^{-1}$ & $<20$ & $20-40$ & $>40$ & & & 150 \\
\hline $\mathrm{Mn}^{1 /}$ & $\mathrm{mg} \mathrm{kg}^{-1}$ & $<5$ & $5-15$ & $>15$ & & & 79 \\
\hline $\mathrm{Zn}^{1 /}$ & $\mathrm{mg} \mathrm{kg}^{-1}$ & $<2$ & $2-7$ & $>7$ & & & 8,5 \\
\hline $\mathrm{B}^{2 /}$ & $m g \mathrm{~kg}^{-1}$ & $<0,5$ & $0,5-1$ & $>1$ & & 0,6 & \\
\hline $\mathrm{MO}^{3 /}$ & $\%$ & $<3$ & $3-5$ & $>5$ & & 3,1 & \\
\hline
\end{tabular}

${ }^{1 / \mathrm{N}}, \mathrm{P}, \mathrm{K}, \mathrm{Ca}, \mathrm{Mg}, \mathrm{Cu}, \mathrm{Fe}, \mathrm{Mn}$ y $\mathrm{Zn}$ fueron determinados con el método de Olsen modificado.

${ }^{2 / S}$ y B fueron determinados con el método del Fosfato de Calcio Monobásico

3/ Titulación de Walkley Black.

ello se cortó la materia verde a $5 \mathrm{~cm}$ por encima del nivel del suelo obtenida en el $\mathrm{m}^{2}$ del cuadrante PVC y luego se registró su peso en $\mathrm{kg}$ con ayuda de una balanza electrónica de precisión; mediante extrapolación se estimó el rendimiento ha-1. Para la determinación del rendimiento en materia seca, se extrajo una alícuota de $200 \mathrm{~g}$ de materia verde, que luego fue llevado a laboratorio y colocado en estufa a $65^{\circ} \mathrm{C}$ por 72 horas hasta alcanzar peso constante, según lo descrito por Sanches et al. (2017). Con los datos de materia seca obtenidos en laboratorio se estimó el rendimiento en $\mathrm{kg}$ $\mathrm{ha}^{-1}$. Con los promedios de rendimiento de materia seca se estimó la eficiencia agronómica del $\mathrm{N}$ aplicado, lo cual se calculó siguiendo la metodología descrita por Yadav et al. (2017).

La eficiencia agronómica (EA) del $\mathrm{N}$ aplicado por hectárea se calculó de acuerdo a IPNI (2012):

$\mathrm{EA}_{\mathrm{N}}=\mathrm{kg}$ de incremento en biomasa por $\mathrm{kg}^{-1}$ de $\mathrm{N}$ aplicado

$\mathrm{EA}_{\mathrm{N}}=\left[\left(\mathrm{RB}_{+\mathrm{N}}-\mathrm{RB}_{0 \mathrm{~N}}\right) / \mathrm{FN}\right]$

donde:

$R B_{+\mathrm{N}}=$ es el rendimiento en biomasa con aplicación de $\mathrm{N}$

$\mathrm{RB}_{0 \mathrm{~N}}=$ es el rendimiento en biomasa $\sin$ aplicación de $\mathrm{N}$

$\mathrm{FN}=$ cantidad de $\mathrm{N}$ aplicado

Los datos fueron sometidos a análisis de varianza. La separación de medias fue calculada con prueba de Tukey al 5\% de probabilidades de error. Además, se realizaron análisis de regresión con el interés de establecer la tendencia de respuesta de la fertilización con $\mathrm{N}$ para ambas frecuencias de corte.

\section{RESULTADOS Y DISCUSIÓN}

La producción de materia seca fue significativamente $(p<0,01)$ influenciada por las frecuencias de corte, los niveles de $\mathrm{N}$ y su respectiva interacción, en los cuatro ciclos de corte consecutivos evaluados (Tabla 2). Se aprecia que la producción de materia seca fue mayor a los 45 días después del corte (DDC), independientemente de los niveles de N. De forma similar se observó que a mayor cantidad de $\mathrm{N}$ la producción de materia seca se incrementa independientemente de las frecuencias de corte, que para este caso los niveles de 150 y $200 \mathrm{~kg} \mathrm{~N} \mathrm{ha}^{-1}$ obtuvieron el mayor rendimiento (Tabla 2). Los datos coinciden con los obtenidos por Borges et al. (2017), quienes reportaron mayor producción de forraje del pasto Cynodon cv. Tifton- 85 con $210 \mathrm{~kg}$ de $\mathrm{N} \mathrm{ha}^{-1}$. Así mismo los resultados se asemejan a los reportados por Taffarel et al. (2016), quienes consiguieron mayor producción de materia seca en el pasto Tiffon-85 con $100 \mathrm{~kg} \mathrm{~N}$ ha-1 $^{-1}$ que fue el mayor nivel de $\mathrm{N}$ evaluado.

Independientemente de los niveles de $\mathrm{N}$ evaluados, la producción de materia seca mostró un incremento promedio de 33,93\% entre los 30 y 45 días de frecuencias. Este hecho sugiere que desde el punto de vista productivo bajo las condiciones del trópico bajo donde se 
Tabla 2. Efecto de cuatro niveles de $\mathrm{N}$ sobre la producción de materia seca en función de dos frecuencias de corte del pasto C. plectostachyus.

Table 2. Effect of four levels of $\mathbf{N}$ application on the production of dry matter as a function of two frequencies of grass cut $C$. plectostachyus.

\begin{tabular}{|c|c|c|c|c|c|c|}
\hline \multirow{2}{*}{ Tratamientos } & & \multicolumn{5}{|c|}{ Producción de materia seca $\left(\mathrm{kg} \mathrm{ha}^{-1}\right)$} \\
\hline & & Corte 1 & Corte 2 & Corte 3 & Corte 4 & Promedio \\
\hline \multicolumn{7}{|c|}{ Efecto de tiempos de crecimiento } \\
\hline $30 \mathrm{DDC}$ & & 2399,40 a & $2372,00 \mathrm{a}$ & $2431,50 \mathrm{a}$ & 2452,00 a & $2413,73 a$ \\
\hline $45 \mathrm{DDC}$ & & $3590,80 \mathrm{~b}$ & $3648,00 \mathrm{~b}$ & $3675,50 \mathrm{~b}$ & $3698,50 \mathrm{~b}$ & $3653,20 \mathrm{~b}$ \\
\hline \multicolumn{7}{|c|}{ Efecto de niveles de $\mathrm{N}$} \\
\hline 0 & & $2666,75 \mathrm{a}$ & $2667,50 \mathrm{a}$ & $2431,50 \mathrm{a}$ & $2681,25 \mathrm{a}$ & $2611,75 \mathrm{a}$ \\
\hline 50 & & $2773,75 \mathrm{a}$ & $2783,75 \mathrm{~b}$ & $3021,25 \mathrm{~b}$ & $2825,00 \mathrm{~b}$ & $2850,94 \mathrm{~b}$ \\
\hline 100 & & $2959,75 \mathrm{~b}$ & $2961,25 c$ & $3246,25 c$ & $3037,50 \mathrm{c}$ & $3051,19 \mathrm{c}$ \\
\hline 150 & & $3123,25 \mathrm{c}$ & $3165,00 \mathrm{~d}$ & $3525,00 \mathrm{~d}$ & $3268,75 \mathrm{~d}$ & $3270,50 \mathrm{~d}$ \\
\hline 200 & & $3452,00 \mathrm{~d}$ & $3472,50 \mathrm{e}$ & $3675,50 \mathrm{e}$ & $3563,75 \mathrm{e}$ & $3540,94 \mathrm{e}$ \\
\hline \multicolumn{7}{|c|}{ Efecto de interacción tiempo de crecimiento $\mathrm{x} N$} \\
\hline \multirow[t]{5}{*}{$30 \mathrm{DDC}$} & 0 & $2127,50 \mathrm{a}$ & $2050,00 \mathrm{a}$ & $2075,00 \mathrm{a}$ & $2100,00 \mathrm{a}$ & $2088,13 \mathrm{a}$ \\
\hline & 50 & $2215,50 \mathrm{ab}$ & $2165,00 \mathrm{a}$ & $2217,50 \mathrm{a}$ & $2230,00 \mathrm{ab}$ & $2207,00 \mathrm{~b}$ \\
\hline & 100 & $2395,00 \mathrm{bc}$ & $2337,50 \mathrm{~b}$ & $2415,00 \mathrm{~b}$ & $2405,00 \mathrm{~b}$ & 2388,13 c \\
\hline & 150 & $2507,50 \mathrm{c}$ & $2537,50 \mathrm{c}$ & $2637,50 \mathrm{c}$ & $2622,50 \mathrm{c}$ & $2576,25 d$ \\
\hline & 200 & $2751,50 \mathrm{~d}$ & $2770,00 \mathrm{~d}$ & $2812,50 \mathrm{~d}$ & $2902,50 \mathrm{~d}$ & $2809,13 \mathrm{e}$ \\
\hline \multirow[t]{5}{*}{$45 \mathrm{DDC}$} & 0 & $3206,00 \mathrm{e}$ & $3285,00 \mathrm{e}$ & $3245,00 \mathrm{e}$ & $3262,50 \mathrm{e}$ & $3249,63 \mathrm{f}$ \\
\hline & 50 & 3332,00 ef & 3402,50 ef & $3412,50 \mathrm{f}$ & $3420,00 \mathrm{f}$ & $3391,75 \mathrm{~g}$ \\
\hline & 100 & $3524,50 \mathrm{f}$ & 3585,00 ef & $3627,50 \mathrm{~g}$ & $3670,00 \mathrm{~g}$ & $3601,75 \mathrm{~h}$ \\
\hline & 150 & $3739,00 \mathrm{~g}$ & $3792,50 \mathrm{f}$ & $3855,00 \mathrm{~h}$ & $3915,00 \mathrm{~h}$ & $3825,38 \mathrm{i}$ \\
\hline & 200 & $4152,50 \mathrm{~h}$ & $4175,00 \mathrm{~h}$ & $4237,50 \mathrm{i}$ & $4225,00 \mathrm{i}$ & $4197,50 \mathrm{j}$ \\
\hline \multicolumn{7}{|c|}{ p-valor ANOVA } \\
\hline Frecuencias & corte & 0,0001 & 0,0001 & 0,0001 & 0,0001 & 0,0001 \\
\hline Niveles N & & 0,0001 & 0,0001 & 0,0001 & 0,0001 & 0,0001 \\
\hline Frecuencias & iveles de $\mathrm{N}$ & 0,0043 & 0,0103 & 0,0034 & 0,0100 & 0,0001 \\
\hline
\end{tabular}

${ }^{1 /}$ DDC: Días después del corte

${ }^{2 /}$ Medias en las columnas con letras distintas difieren significativamente de acuerdo al test de Tukey al 5\% de probabilidades de error.

desarrolló el experimento, conviene realizar el corte o defoliación del pasto cada 45 días, aunque posiblemente se pueda sacrificar valor nutricional. Este hallazgo coincide a los reportes de Taffarel et al. (2016) quienes evaluaron intervalos de rebrote de 28 y 35 días en el pasto Tiffon-85, donde obtuvieron mayor acumulación de materia seca con la mayor frecuencia de defoliación. En este mismo contexto resultados hallados por Timpong et al. (2015) indicaron que la producción de materia seca del pasto $C$. nlemfuensis se incrementó significativamente a frecuencias de defoliación más amplios.

Independientemente de las frecuencias de corte evaluados, la materia seca se incrementó en promedio 8,$39 ; 14,40 ; 20,14$ y $26,24 \%$ con 50 ,
100,150 y $200 \mathrm{~kg} \mathrm{~N} \mathrm{ha}^{-1}$, con respecto a la parcela con omisión de $\mathrm{N}$. Lo anterior sugiere que el pasto C. plectostachyus responde positivamente a la fertilización nitrogenada y que el $\mathrm{N}$ es un nutriente clave para la producción de forraje, dado que este elemento está involucrado en la estructuración de tejidos, órganos y desarrollo de las plantas a través de mecanismos moleculares (Shah et al., 2016; Ueda et al., 2017).

La Fig. 1 muestra la tendencia lineal de la producción de MS en ambas frecuencias de corte y en función de niveles crecientes de N. En el caso de MS el modelo de regresión lineal predice una

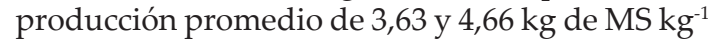
de $\mathrm{N} \mathrm{ha}^{-1}$ aplicado a los 30 y 45 días después del corte (Fig. 1). 


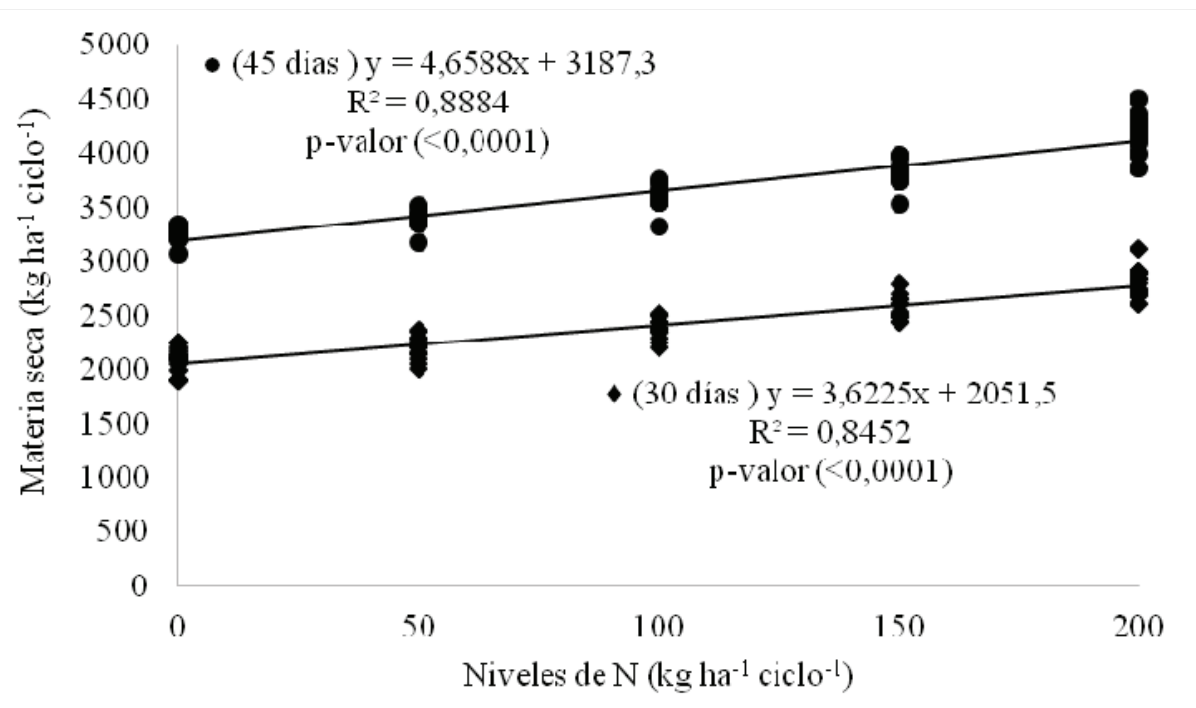

Fig. 1. Producción de materia seca a los $30(\diamond)$ y $45(\bullet)$ días del corte del pasto $C$. plectostachyus en función de niveles crecientes de $\mathrm{N} \mathrm{ha}^{-1}$.

Fig. 1. Production of dry matter $30(\diamond)$ and $45(\bullet)$ days after the cut of the $C$. plectostachyus grass based on increasing levels of $\mathrm{N} \mathrm{ha}^{-1}$.

La eficiencia agronómica del nitrógeno (EAN) fue significativa influenciada $(\mathrm{p}<0,01)$ por las frecuencias de corte y los niveles de nitrógeno evaluados en los cuatro ciclos de corte realizados, mientras que la interacción entre frecuencia de corte $x$ niveles de nitrógeno no influyó estadísticamente $(p>0,05)$ sobre la eficiencia agronómica del nitrógeno en base a materia seca (Tabla 3). Lo anterior denota que la eficiencia agronómica está en función de la cantidad individual de nitrógeno aplicado y del incremento de la producción de biomasa (IPNI, 2012; Yadav et al., 2017). En todos los ciclos de corte evaluados la eficiencia agronómica determinada en base a materia seca se incrementó con la frecuencia de 45 días y con los niveles crecientes de nitrógeno, aunque en los cortes 3 y 4, así como para el promedio de los cuatro cortes, la EAN fue estadísticamente igual entre los niveles de 150 y $200 \mathrm{~kg} \mathrm{~N} \mathrm{ha}^{-1}$ (Tabla 3).

En promedio la EAN en base a materia seca se incrementó $18,18 \%$ con la frecuencia de corte de 45 días, respecto a la de 30 días. Por su parte, la EAN en base materia seca de los niveles crecientes de $\mathrm{N}$ en promedio se incrementó 18,$81 ; 24,23$ y $34,93 \%$ con 100,150 y $200 \mathrm{~kg} \mathrm{~N} \mathrm{ha}^{-1}$, respectivamente, en relación a al nivel más bajo de $\mathrm{N}$ aplicado (Tabla 3).

Los resultados de rendimiento en base a materia seca, se corresponden a los hallados por Taffarel et al. (2016) y Taffarel et al. (2017) quienes reportaron mayor rendimiento de materia seca en Cynodon spp. cv. Tifton 85 a medida que se incrementaron los niveles de $\mathrm{N}$ de $0,25,50,75$ y $100 \mathrm{~kg} \mathrm{ha}^{-1}$ de $\mathrm{N}$, lo cual se correspondió con una mayor concentración de proteína cruda. Por su parte, Borges et al. (2017) alcanzaron mayor acumulación de biomasa seca en el mismo genotipo de pasto con el aumento de niveles crecientes de nitrógeno de 0, 60, 120, 180 y 240 $\mathrm{kg} \mathrm{ha}^{-1}$ de $\mathrm{N}$, lo cual se relacionó a mayores tasas de índices de área foliar y radiación fotosintéticamente activa interceptada.

La eficiencia agronómica de $\mathrm{N}$ obtenidas se contraponen a los resultados reportados por Silveira et al. (2007) y Schneider et al. (2019) quienes reportaron que la eficiencia de la fertilización nitrogenada tiende a disminuir a medida que se incrementan las tasas de $\mathrm{N}$. Sin embargo, los resultados se asemejan a los reportados por Sigua et al. (2013), quienes reportaron mayor eficiencia agronómica en Panicum hematomon y Paspalum notatum cuando las dosis de $\mathrm{N}$ se incrementó de 100 a $200 \mathrm{~kg} \mathrm{ha}^{-1}$ en suelos inundados. Resultados similares fueron hallados por Oliveira et al. (2014) que alcanzaron mayor eficiencia agronómica con dosis crecientes de $0,60,120,180,240 \mathrm{~kg} \mathrm{~N}^{-1}$ en pasto Brachiaria brizantha cv. Marandu. Por su parte, Assmann et al. (2018) reportaron resultados variables en cuanto a uso eficiente de $\mathrm{N}$ en Cynodon spp. cv. Tifton 85, dado que durante la temporada 20112012 la eficiencia del N se incrementó cuando las dosis aumentaron de 0, 100, 200 y $300 \mathrm{~kg}$ de N $\mathrm{ha}^{-1}$, mientras se dio lo contrario en la temporada 2012-2013.

La respuesta lineal de la producción de biomasa y eficiencia agronómica de $\mathrm{N}$ del pasto 
Tabla 3. Eficiencia agronómica de $\mathbf{N}$ cuantificada en base a materia seca de acuerdo a dos frecuencias de corte y cuatro niveles de fertilización nitrogenada en $C$. plectostachyus.

Table 3. Agronomic efficiency of $\mathrm{N}$ quantified based on dry matter according to two cutoff frequencies and four levels of nitrogen fertilization in C. plectostachyus.

\begin{tabular}{|c|c|c|c|c|c|}
\hline \multirow{2}{*}{ Tratamientos } & \multicolumn{5}{|c|}{ Eficiencia agronómica de $\mathbf{N}$ en base a Materia seca } \\
\hline & Corte 1 & Corte 2 & Corte 3 & Corte 4 & Promedio \\
\hline \multicolumn{6}{|c|}{ Efecto de tiempos de crecimiento } \\
\hline $30 \mathrm{DDC}^{1 /}$ & $2,52 a^{2 /}$ & $3,01 \mathrm{a}$ & $3,42 \mathrm{a}$ & $3,29 \mathrm{a}$ & 3,06 a \\
\hline $45 \mathrm{DDC}$ & $3,50 \mathrm{~b}$ & $3,30 \mathrm{~b}$ & $4,05 \mathrm{~b}$ & $4,10 \mathrm{~b}$ & $3,74 b$ \\
\hline \multicolumn{6}{|c|}{ Efecto de niveles de $\mathrm{N}$} \\
\hline 0 & ----- & ----- & ----- & ----- & ----- \\
\hline 50 & $2,14 \mathrm{a}$ & $2,33 \mathrm{a}$ & 3,10 a & 3,29 a & $2,72 \mathrm{a}$ \\
\hline 100 & $2,93 \mathrm{~b}$ & $2,94 \mathrm{~b}$ & $3,61 \mathrm{ab}$ & $3,92 \mathrm{ab}$ & $3,35 \mathrm{~b}$ \\
\hline 150 & $3,04 \mathrm{~b}$ & $3,32 b$ & $3,91 \mathrm{~b}$ & $4,10 \mathrm{ab}$ & $3,59 \mathrm{~b}$ \\
\hline 200 & $3,93 \mathrm{c}$ & $4,03 \mathrm{c}$ & $4,33 \mathrm{~b}$ & $4,41 \mathrm{ab}$ & $4,18 \mathrm{bc}$ \\
\hline $\mathrm{CV}(\%)$ & 18,58 & 11,26 & 14,68 & 17,27 & 7,82 \\
\hline \multicolumn{6}{|l|}{ p-valor ANOVA } \\
\hline Frecuencias de corte & 0,0001 & 0,0018 & 0,0039 & 0,0017 & 0,0001 \\
\hline Niveles N & 0,0001 & 0,0001 & 0,0018 & 0,0008 & 0,0001 \\
\hline Frecuencias $x$ niveles de $\mathrm{N}$ & 0,2621 & 0,1130 & 0,3095 & 0,9007 & 0,0701 \\
\hline
\end{tabular}

C. plectostachyus a las dosis crecientes de N, puede estar favorecida por el bajo contenido del elemento en el suelo reportado por el análisis de fertilidad inicial previo al establecimiento del experimento (Tabla 1), dado que según estudios, la respuesta de los cultivos a la fertilización nitrogenada es mayor cuando el suelo es deficiente en $\mathrm{N}$, además que la mejora del uso eficiente del nutriente se incrementa con la absorción de $\mathrm{N}$ por la planta (Lammerts y Struik, 2017). En este sentido, el incremento en uso eficiente de $\mathrm{N}$ puede deberse a una mayor necesidad de la planta por el elemento bajo las condiciones donde se desarrolló el experimento, dada las funciones estructurales y metabólicas que desempeña el nutriente, pues se conoce que el nitrógeno es componente básico de aminoácidos, proteínas y hormonas, que conforman el tejido básico de membranas y paredes celulares, así como también de enzimas y pigmentos fotosintéticos responsables de la absorción de luz y producción de fotoasimilados (Ueda et al., 2017; Borges et al., 2017; Yasuoka et al., 2017).

De acuerdo al rendimiento de biomasa y a la eficiencia agronómica obtenida en esta investigación, bajo las condiciones del trópico bajo del cantón Chone, se puede sugerir que la fertilización nitrogenada del pasto estrella puede ser suficiente con las dosis de $150 \mathrm{~kg}$ de $\mathrm{N} \mathrm{ha}^{-1}$, dado que el incremento de MS entre 150 y $200 \mathrm{~kg}$
$\mathrm{N} \mathrm{ha}^{-1}$ es apenas del $6 \%$ y la eficiencia agronómica del $\mathrm{N}$ entre ambas dosis fue estadísticamente igual. Sin embargo, las dosis deberían ajustarse en el futuro desde un punto de vista económico y de calidad bromatológica, dado que en esta investigación estos dos aspectos no fueron considerados.

\section{CONCLUSIONES}

El pasto C. plectostachyus mostró respuestas positivas a la fertilización nitrogenada bajo las condiciones de suelo y clima donde se desarrolló el estudio.

La producción de materia seca se incrementó linealmente con las dosis de $\mathrm{N}$ en ambas frecuencias de corte.

La frecuencia de corte de 45 días mostró la mayor producción de materia seca.

La fertilización del pasto estrella bajo las condiciones del trópico bajo de Chone puede ser suficiente con $150 \mathrm{~kg}$ de $\mathrm{N} \mathrm{ha}^{-1}$.

\section{RECONOCIMIENTO}

Dedicado a la memoria de nuestro amigo, colega y compañero Dídimo Efraín Vera Arteaga, quien por razones que solo Dios conoce dejo este mundo prematuramente. Sin embargo, se 
reconoce su contribución al desarrollo académico universitario y a la investigación científica del país.

\section{LITERATURA CITADA}

Assmann, T., E. Sonego, A. Assmann, P. Adami, and C. Cuzzi. 2018. Effect of splitting nitrogen fertilization on Tifton 85: Yield, nitrogen use efficiency, and nitrogen nutritional status of plants and soil. African Journal of Agricultural Research 13(22):1154-1162. doi. 10.5897/AJAR2017.12869.

Barhoumi, Z. 2017. Insights into the growth response and nitrogen accumulation and use efficiency of the Poaceae grass Brachypodium distachyon to high nitrogen availability. Russian Journal of Plant Physiology 64(6):839-844. doi.org/10.1134/ S1021443717060024

Borges, B., M. Silveira, S. Cardoso, E. Moline, A. Coutinho, F. Lucas, et al. 2017. Growth, herbage accumulation, and nutritive value of 'Tifton 85 ' bermudagrass as affected by nitrogen fertilization strategies. Crop Sci. 57(6):3333-3342. doi.org/10.2135/ cropsci2016.10.0890.

Bonifaz, N., R. León, y F. Gutiérrez. 2018. Pastos $\mathrm{y}$ forrajes del Ecuador: Siembra y producción de pasturas. Editorial Universitaria AbyaYala, Universidad Politécnica Salesiana, Quito, Ecuador. 616 p. ISBN 978-9978-10-3180. Disponible en: file://C:/Users/GALO $\% 20$ CEDE\%C3\%910/Downloads/Pastos\%20 y\%20forrajes\%20del\%20Ecuador\%20(1).pdf (Consulta 25 mayo 2019).

Castillo, M. 2014. Análisis de la productividad y competitividad de la ganadería de carne en el litoral ecuatoriano (Resultados de Consultoría para RIMISP-Parte I). Serie Documentos de Trabajo No 144. Grupo de Trabajo: Desarrollo con Cohesión Territorial. Programa Cohesión Territorial para el Desarrollo. RIMISP, Santiago, Chile. Disponible en: http://www.rimisp.org/wpcontent/ files_mf/1431715949GanaderiadeCarneDoc. deResultados_Final_editado.pdf (Consulta 24 mayo 2019).

Cormier, F., J. Foulkes, B. Hirel, D. Gouache, Y. Moënne-Loccoz, and J. Le Gouis. 2016. Breeding for increased nitrogen-use efficiency: a review for wheat (T. aestivum L.). Plant Breeding 1-24. doi:10.1111/pbr.12371.

Cristiano, P., G. Posse, and C. Di Bella. 2015. Total and aboveground radiation use efficiency in $\mathrm{C}^{3}$ and $\mathrm{C}^{4}$ grass species influenced by nitrogen and water availability. Grassland Science 61(3):131-141. doi.org/10.1111/grs.12086.
Han, M., M. Okamoto, P. Beatty, S. Rothstein, and A. Good. 2015. The genetics of nitrogen use efficiency in crop plants. Annu. Rev. Genet. 49:9.1-9.21. doi: 10.1146/annurevgenet-112414-055037.

IPNI. 2012. 4R Plant Nutrition Manual: A Manual for Improving the Management of Plant Nutrition. T.W. Bruulsema, P.E. Fixen, G.P. Sulewski. (eds.) International Plant Nutrition Institute, Norcross, Georgia, USA.

Hirel, B., T. Tétu, P. Lea, and F. Dubois. 2011. Improving nitrogen use efficiency in crops for sustainable agriculture. Sustainability 3(9):1452-1485. doi.org/10.3390/su3091452.

Lammerts, T., and P. Struik. 2017. Diverse concepts of breeding for nitrogen use efficiency. A review. Agron. Sustain. Dev. 37(50): doi.org/10.1007/s13593-017-0457-3.

López, F., J. Estrada, F. Avilés, G. Yong, P. Hernández, R. Martínez, et al. 2010. Agronomic evaluation and chemical composition of african star grass (Cynodon plectostachyus) in the southern region of the state of Mexico. Tropical and Subtropical Agroecosystems. 12(1):151-159. E-ISSN: 1870-0462.

Li, H., B. Hu, and C. Chu. 2017. Nitrogen use efficiency in crops: lessons from Arabidopsis and rice. Journal of Experimental Botany 68(10): 2477-2488. doi: 10.1093/jxb/erx101.

MAE. 2013. Sistema de Clasificación de los Ecosistemas del Ecuador Continental. Subsecretaría de Patrimonio Natural. Quito. 232 p. Ministerio de Ambiente del Ecuador. Disponible en http://app.sni.gob.ec/sni-link/ sni/PDOT/NIVEL\%20NACIONAL/MAE/ ECOSISTEMAS/DOCUMENTOS/Sistema. pdf. (Consulta 28 junio 2019)

MAGAP. 2016. La política agropecuaria ecuatoriana: hacia el desarrollo territorial rural sostenible: 2015-2025 I Parte. MAGAP, Quito, Ecuador. 480 p. ISBN: 978-9942-22019-6. Ministerio de Agricultura, Ganadería, Acuacultura y Pesca. Disponible en: http:// servicios.agricultura.gob.ec/politicas/La $\% 20$ Pol\%C3\%ADticas\%20Agropecuarias\%20 al\%20\%202025\%20I\%20parte.pdf (Consulta 25 mayo de 2019).

Mohan, K., H. Hanumanthappa, S. Marimuthu, and C. Meenambigai. 2018. A review on enhancing the fertilizers use efficiency to minimize environmental impacts. International Journal of Chemical Studies 6(3):2167-2174. E-ISSN: 2321-4902. 
Moreno, J., G. Sevillano, O. Valverde, V. Loayza, R. Haro, and J. Zambrano. 2018. Soil from the Coastal Plane. Chapter 2. p. 27-77. In Espinosa, J., J. Moreno, and G. Bernal (eds.). The Soils of Ecuador. Springer International Publishing AG 2018. doi.org/10.1007/978-3319-25319-0.

Oliveira, T., F. Leonel, J. Pereira, C. Silva, D. Ferreira, and J. Zervoudakis. 2014. Yield, chemical composition, and efficiency of use of nitrogen by Marandu grass. R. Bras. Zootec., 43(8):440-444. doi.org/10.1590/ S1516-35982014000800007.

Pereira, O., R. Rovetta, K. Ribeiro, M. Santos, D. Fonseca, y P. Cecon. 2011. Características morfogênicas e estruturais do capimtifton 85 sob doses de nitrogênio e alturas de corte. Revista Brasileira de Zootecnia, Viçosa, MG. 40(9):1870-1878.

Prasad, R. 2013. Fertilizer Nitrogen, Food Security, Health and the Environment. Proc. Indian Natn. Sci. Acad. 79(4):997-1010.

Rashid, G., M. Avais, S. Saleem, M. Hassan, R. Ahmed, M. Ali, et al. 2018. Influence of nitrogen fertilizer on nitrate contents of plants: A prospective aspect of nitrate poisoning in dairy animals. Pakistan Journal of Zoology 51(1):249-253. doi: 10.17582/ journal.pjz/2019.51.1.249.255.

Rütting, T., H. Aronsson, and S. Delin. 2018. Efficient use of nitrogen in agriculture. Nutr. Cycl. Agroecosyst. 110(1):1-5. doi. org/10.1007/s10705-017-9900-8.

Sanches, A., E. Gomes, M. Rickli, E. Friske, and J. Fasolin. 2017. Productivity and nutritive value of Tifton 85 in summer, with and without irrigation under different nitrogen doses. Eng. Agric. 37(2):246256. doi.org/10.1590/1809-4430-eng.agric. v37n2p246-256/2017.

Shah, J., A. Niaz, M. Ghulam, H. Abdul, M.B. Ghulam, H. Khalid, et al. 2016. Role of nitrogen for plant growth and development: A review. Advances in Environmental Biology 10(9):209-218. ISSN-1995-0756.

Sharma, L., and S. Bali. 2017. A review of methods to improve nitrogen use efficiency in agriculture. Sustainability 10(51):1-23. doi:10.3390/su10010051.

Silveira, M., V. Haby, and A. Leonard. 2007. Response of coastal bermudagrass yield and nutrient uptake efficiency to nitrogen sources. Agronomy Journal 99(3):707-714. doi:10.2134/agronj2006.0200.
Sigua, G., M. Williams, C. Chase, J. Grabowski, and M. Kongchum. 2013. Nitrogen recovery and agronomic efficiency of forages with nitrogen fertilization under flooded condition. Agricultural Sciences 4(3):138-148. doi.org/10.4236/as.2013.43021.

Schneider, R., K. Chekhovskiy, P. Muñoz, S. Kwon, and M. Saha. 2019. Characterization of bermudagrass (Cynodon dactylon L.) germplasm for nitrogen use efficiency. Euphytica 215(40):1-14. doi.org/10.1007/ s10681-019-2347-z.

Taffarel, L., E. Mesquita, D. Castagnara, S. Galbeiro, P. Costa, and P. De Oliveira. 2016. Tifton 85 grass responses to different nitrogen levels and cutting Intervals. Ciências Agrárias, Londrina. 37(4):2067-2084. doi.org/10.5433/1679-0359.2016v37n4p2067.

Taffarel, L., E. Mesquita, D. Castagnara, P. Costa, and P. Rabello. 2017. Evaluation of 'Tifton $85^{\prime}$ during hay production using different nitrogen fertilization rates and dehydration methods. African Journal of Agricultural Research 12(40):2995-3004. doi: 10.5897/ AJAR2017.12383.

Timpong, E., L. Adjorlolo, and R. Ayizanga. 2015. The impact of harvest frequency on herbage yield and quality of Cynodon nlemfuensis. West African Journal of Applied Ecology 23(2):7-15.

Ueda, Y., M. Konishi, and S. Yanagisawa. 2017. Molecular basis of the nitrogen response in plants. Soil Science and Plant Nutrition 63(4):329-341. doi:10.1080/00380768.2017.136 0128.

Valles, B., E. Castillo, y H. Bernal. 2016. Rendimiento y degradabilidad ruminal de materia seca y energía de diez pastos tropicales cosechados a cuatro edades. Revista Mexicana de Ciencias Pecuarias 7(2):141 - 158.

Villalobos, L., y J. Arce. 2013. Evaluación agronómica y nutricional del pasto estrella africana (Cynodon nlemfuensis) en la zona de Monteverde, Puntarenas, Costa Rica. I. Disponibilidad de biomasa y fenología. Agronomía Costarricense 37(1):91-101. ISSN 0377-9424.

Villalobos, L., y J. Arce. 2014. Evaluación agronómica y nutricional del pasto estrella africana (Cynodon nlemfuensis) en la zona de Monteverde, Puntarenas, Costa Rica. II. Valor nutricional. Agronomía Costarricense 38(1):133-145. ISSN 0377-9424. 
Yadav, M., R. Kumar, C. Parihar, R. Yadav, S. Jat, H. Ram, et al. 2017. Strategies for improving nitrogen use efficiency: A review. Agricultural Reviews 38(1):29-40. doi:10.18805/ag.v0iOF.7306.

Yasuoka, J., C. Pedreira, V. da Silva, A. Alonso, L. da Silva, and F. Gomes. 2017. Canopy height and $\mathrm{N}$ affect herbage accumulation and the relative contribution of leaf categories to photosynthesis of grazed brachiariagrass pastures. Grass and Forage Science 73(1): 183-192. doi.org/10.1111/gfs.12302.

Yong, G., V. Pires, F. Avilés, and O. Castelán. 2012. Modeling growth of star grass (Cynodon plectostachyus) in the subtropical regions of central Mexico. Tropical and Subtropical Agroecosystems 15:273-300.
Yong, G., V. Pires, F. Avilés., and O. Castelán. 2014. Simulating the effect of climate on star grass growth and quality and its capacity to support milk production in the tropical regions of Mexico. Ciência Rural, Santa Maria 44(8):1486-1493. doi.org/10.1590/0103$8478 \mathrm{cr} 20130987$.

Zhao, H., X. Li, and Y. Jiang. 2019. Response of nitrogen losses to excessive nitrogen fertilizer application in intensive greenhouse vegetable production. Sustainability 11(1513):1-15. doi:10.3390/su11061513. 